OPEN ACCESS

Edited by:

Hauke Busch,

University of Lübeck, Germany

Reviewed by:

Geoffroy Andrieux,

Universität Freiburg, Germany

Chai K. Lim,

Macquarie University, Australia

*Correspondence:

Joo-Youn Cho

joocho@snu.ac.kr

${ }^{\dagger}$ These authors have contributed equally to this work

¥Present address:

Da Jung Kim,

Metabolomics Core Facility,

Department of Transdisciplinary

Research and Collaboration,

Biomedical Research Institute, Seoul

National University Hospital, Seoul,

South Korea

Specialty section:

This article was submitted to

Pulmonary Medicine,

a section of the journal

Frontiers in Medicine

Received: 19 October 2020 Accepted: 12 April 2021

Published: 14 May 2021

Citation:

Kim DJ, Oh JY, Rhee CK, Park SJ,

Shim JJ and Cho J-Y (2021) Metabolic Fingerprinting Uncovers the

Distinction Between the Phenotypes

of Tuberculosis Associated COPD and

Smoking-Induced COPD.

Front. Med. 8:619077.

doi: 10.3389/fmed.2021.619077

\section{Metabolic Fingerprinting Uncovers the Distinction Between the Phenotypes of Tuberculosis Associated COPD and Smoking-Induced COPD}

\author{
Da Jung Kim ${ }^{1,2+\neq}$, Jee Youn $\mathrm{Oh}^{3 \dagger}$, Chin Kook Rhee ${ }^{4}$, Seoung Ju Park ${ }^{5}$, Jae Jeong Shim ${ }^{3}$ \\ and Joo-Youn Cho ${ }^{1,6 *}$
}

${ }^{1}$ Department of Clinical Pharmacology and Therapeutics, Seoul National University College of Medicine and Hospital, Seoul, South Korea, ${ }^{2}$ Seoul National University Medical Research Center, Seoul National University College of Medicine and Hospital, Seoul, South Korea, ${ }^{3}$ Division of Pulmonary, Allergy and Critical Care Medicine, Department of Internal Medicine, Korea University Guro Hospital, Seoul, South Korea, ${ }^{4}$ Division of Pulmonary Medicine, Department of Internal Medicine, Catholic University Seoul Hospital, Seoul, South Korea, ${ }^{5}$ Division of Pulmonary Medicine, Department of Internal Medicine, Chonbuk National University Hospital, Jeonju, South Korea, ${ }^{6}$ Department of Biomedical Sciences, Seoul National University College of Medicine, Seoul, South Korea

Background: Although smoking is considered the main cause of chronic obstructive pulmonary disease (COPD), several other risk factors, including pulmonary tuberculosis (TB), contribute significantly to disease causation, particularly in developing countries. However, the underlying pathogenesis of TB-associated COPD (T-COPD) is unclear. Moreover, the need for prompt diagnosis and treatment of T-COPD to decrease the future burden of inflammation is underestimated. This study aimed to identify distinctive endogenous metabotypes of T-COPD, compared to smoking-associated COPD (S-COPD).

Methods: Cross-sectional metabolomic analyses and clinical examinations of serum samples were performed for three groups of 168 male subjects: T-COPD $(n=59)$, S-COPD $(n=70)$, and healthy normal controls $(n=39)$. To retain a broad spectrum of metabolites, we performed technically distinct analyses (global metabolomic profiling using LC-QTOFMS and targeted analyses using LC-MS/MS).

Results: Higher levels of IL-6 and C-reactive protein and St. George Respiratory Questionnaire scores were seen in the T-COPD group, compared to those in the S-COPD group. Global metabolomic profiling showed elevated metabolites, including arachidonic and eicosanoic acids, in the T-COPD group. Typical changes in tryptophan catabolism were observed through targeted profiling. Additionally, in the T-COPD group, kynurenine was elevated, and serotonin levels were reduced; therefore, indoleamine dioxygenase (IDO)/tryptophan hydroxylase (TPH) activities were dysregulated. Correlation analyses showed that changes in oxylipins were positively correlated with serum levels of IL-6 and C-reactive protein. 
Conclusion: Patients with TB-related COPD have enhanced inflammatory responses that may be linked to fatty acid pathways and tryptophan catabolism, which could be novel therapeutic targets for T-COPD.

Keywords: chronic obstructive pulmonary disease, tuberculosis, metabolomics, biomarker, smoking

\section{INTRODUCTION}

Chronic obstructive pulmonary disease (COPD) is a major global health problem that causes airflow limitation and increases patient vulnerability to exacerbations and serious inflammatory responses (1). Apart from smoking, several other risk factors, including biomass fuel smoke exposure, childhood lower respiratory tract infections, outdoor air pollution, and treated cases of pulmonary tuberculosis (TB) play a significant role in disease etiology, particularly in developing countries (2). Approximately 10 million people are infected with TB annually, and pulmonary system injury is the major effect of TB (3). Appropriate treatment can effectively cure TB. However, permanent COPD occurring after eradication of the organism is seen in more than $50 \%$ of the infected patients $(3,4)$. TB-associated COPD (T-COPD) develops at a younger age compared with smoking-associated COPD (S-COPD) (5-7). However, prompt diagnosis and treatment of T-COPD based on an understanding of the pathogenesis to decrease the future burden of chronic airflow obstruction and inflammation are seldom achieved (8).

T-COPD was previously considered a static disease causing only parenchymal destruction, and extensive research on the treatment and control of morbidities in these patients have not been conducted to the same extent as with SCOPD. However, lung remodeling and chronic inflammatory responses have recently been shown to persist in these patients after treatment completion $(9,10)$. The pathogenesis and inflammatory properties of T-COPD need to be investigated and compared with those of S-COPD, to evaluate the need for the treatment and management of T-COPD.

Global profiling of metabolites in biofluids and tissues has been popularized for identifying the underlying mechanisms, developing disease-specific or grade-specific biomarkers, and assessing therapeutic effects (11-13). The identification of numerous endogenous metabolites has been used to characterize patients with pulmonary diseases, and endogenous metabotyping is a useful technique to differentiate the characteristic features of diseases with very similar phenotypes, such as asthma and chronic airway obstructive disease $(11,14)$. In this study, we used metabolomics to characterize patients with S-COPD and T-COPD, and we investigated the associations of various metabolites with these disease groups.

\section{METHODS}

\section{Study Population}

This multi-center cohort study was performed to identify clinically useful biological markers in chronic airway diseases for the diagnosis and assessment of treatment response, conducted at the Korea University Guro Hospital, Seoul St. Mary's Hospital, and Chonbuk National University Hospital. The study protocol was approved by the institutional review boards (Korea University Guro Hospital: KUGH 13246; Seoul St. Mary's Hospital: KC15OIMI0553; and Chonbuk National University Hospital: 2015-01-018-005), and it fulfilled the tenets of the Declaration of Helsinki. The patients provided written informed consent.

The inclusion criteria for patients with T-COPD were: (1) history of $\mathrm{TB}$ with no change in the chest images over the past year; (2) at least one finding of destroyed pulmonary parenchyma on chest images (lung volume loss, bronchovascular distortion, fibrosis, or secondary bronciectasis), with the total volume of all lesions being greater than one-third of one lung, confirmed by a radiologist or pulmonologist; (3) airflow limitation (postbronchodilator spirometry with forced expiratory volume in 1 second $\left[\mathrm{FEV}_{1}\right] /$ forced vital capacity $\left.[\mathrm{FVC}]<70 \%\right)$ and no history of asthma or COPD before the diagnosis of TB; and (4) no history of respiratory infections within the previous 6 weeks.

S-COPD was diagnosed according to the American Thoracic Society and Global Initiative for Chronic Obstructive Lung Disease guidelines (post-bronchodilator spirometry with $\left.\mathrm{FEV}_{1} / \mathrm{FVC}<70 \%\right)$. Patients with a smoking history of $>10$ packs per year were enrolled, and patients with bronchiectasis, sequelae from a prior TB infection, interstitial lung disease, or other diseases causing airflow obstruction, were excluded.

Blood samples and data from healthy normal controls (NC) with no history of COPD or respiratory symptoms, who had visited for general health assessments, were provided by the Biobank of Korea University Guro Hospital (KU Guro Gene Bank 2018-026). Data on age, sex, body mass index (BMI), and smoking status were collected. For patients with S-COPD and TCOPD, the variables collected at baseline were smoking amount, BMI, symptom scores, results of pulmonary function tests, rate of decline in $\mathrm{FEV}_{1}$, acute exacerbation history, and chest imaging data. Detailed protocol of measurements of clinical parameters is available in the Supplementary Material.

\section{Global Metabolomics Profiling Using LC-QTOF-MS}

The frozen samples of plasma and urine were briefly diluted with an acetonitrile:methanol:water (3:3:4) mixture (HPLC-grade, Millipore, Bedford, MA, USA) and 10\% methanol, respectively. Each sample $(5 \mu \mathrm{L})$ was loaded onto an Acquity UPLC BEH C18 $(1.7 \mu \mathrm{m}, 2.1 \mathrm{~mm} \times 100 \mathrm{~mm}$; Waters Corp., Milford, MA, USA) column and analyzed using an Agilent 6530 QTOF mass spectrometer (Agilent Technologies). The experimental procedure is described in detail in our previous studies $(12,15)$. 
All samples were examined simultaneously in a single run to avoid the batch effect and the overall quality of the analysis procedure was monitored using repeat extracts of a pooled plasma sample.

\section{Targeted Metabolomics Using LC-MS/MS}

The AbsoluteIDQ ${ }^{\circledR}$ p180 kit with an LC-MS/MS system was used to quantify 188 metabolites, allowing the concurrent highthroughput detection and quantification of metabolites in serum samples. The sample preparation and LC-MS/MS analytical procedure are described in detail in our previous reports (13). The average \% coefficient of variance of analytes ranged between $15-30 \% \mathrm{CV}$ range and the concentrations of limit of detection are listed in Supplementary Table 1.

\section{Statistical Analysis}

Clinical data were presented as the median and interquartile range for continuous variables and as a percentage for categorical variables. Data were compared using the Mann-Whitney $U$-test or Kruskal-Wallis test for continuous variables, and Pearson's $\chi^{2}$ test or Fisher's exact test for categorical variables. ANCOVA was used to compare age, BMI, smoking and underlying diseases for IL-6 and CRP. Statistical significance was defined as $P<$ 0.05 . All statistics were analyzed using SPSS 20.0 software (IBM, Chicago, Illinois, USA). We performed logistic regression of discrimination of T-COPD and S-COPD for targeted metabolites. Variables with a $P<0.05$ on univariate analysis and demographic factors including age, BMI, underlying diseases and smoking history were analyzed using multivariate logistic regression analysis. ROC analysis was done for significant metabolites by multivariate logistic regression.

Statistical analyses for the untargeted and targeted metabolic data and network analysis for visualization are available in the Supplementary Material.

\section{RESULTS}

\section{Patient Demographics}

The study included 168 patients (T-COPD, $n=59$; S-COPD, $n=70 ; \mathrm{NC}, n=39)$. The median age of NCs was 55.0 years (50.0-65.0 years), and the median BMI, 23.7 (22.326.1) (Supplementary Table 2). Table 1 shows the baseline characteristics of patients with T-COPD and S-COPD. The patient-reported scores for symptoms and quality of life were similar in both groups, except for the St. George Respiratory Questionnaire (SGRQ) score, which was higher in the T-COPD group than in the S-COPD group. The rate of $\mathrm{FEV}_{1}$ decline and the percentage of acute exacerbations did not show significant differences among the groups. C-reactive protein (CRP) levels, erythrocyte sedimentation rate (ESR), and IL-6 levels were significantly higher in patients with T-COPD than in those with S-COPD (Table 1).

\section{Metabotyping of Patients: Global and Targeted Metabolic Profiling}

The metabolic profiling workflow is shown in Figure 1. We used two different techniques to obtain a broad range of
TABLE 1 | Demographics of patients with S-COPD and T-COPD.

\begin{tabular}{|c|c|c|c|}
\hline & S-COPD $(N=70)$ & T-COPD $(N=59)$ & $P$-value \\
\hline Age, years & $66.0(59.0-72.5)$ & $68.0(64.0-73.0)$ & 0.165 \\
\hline Sex, male & $70(100)$ & $59(100)$ & - \\
\hline $\mathrm{BMI}, \mathrm{kg} / \mathrm{m}^{2}$ & $23.1(21.1-25.9)$ & $22.9(21.1-25.5)$ & 0.519 \\
\hline Smoking amount (pack-year) & $40.0(30.0-50.0)$ & $0.0(0.0-30.0)$ & $<0.001$ \\
\hline \multicolumn{4}{|l|}{ Comorbidities } \\
\hline Heart disease & 27 (38.6) & $25(42.4)$ & 0.661 \\
\hline Cerebrovascular disease & 9 (12.9) & $5(8.5)$ & 0.425 \\
\hline Depression & $2(2.9)$ & $1(1.7)$ & 0.663 \\
\hline \multicolumn{4}{|l|}{ Pulmonary function tests } \\
\hline $\mathrm{FEV}_{1} \%$ predicted & $62.5(45.8-71.0)$ & $54.0(45.0-73.0)$ & 0.496 \\
\hline FVC \% predicted & $84.0(74.0-93.3)$ & $78.0(66.0-89.0)$ & 0.089 \\
\hline $\mathrm{FEV}_{1} / \mathrm{FVC} \%$ predicted & $54.5(41.0-62.0)$ & $53.0(42.0-65.0)$ & 0.465 \\
\hline DLCo \% & $80.5(64.8-97.0)$ & $75.0(62.0-87.0)$ & 0.180 \\
\hline \multicolumn{4}{|l|}{ Symptom scores } \\
\hline $\mathrm{mMRC}$ & $1.0(0.0-1.0)$ & $1.0(0.0-1.0)$ & 0.654 \\
\hline CAT & $14.0(10.0-19.0)$ & $17.0(11.0-21.0)$ & 0.096 \\
\hline SGRQ & $16.9(9.0-29.2)$ & $22.1(13.8-34.5)$ & 0.048 \\
\hline $\mathrm{FEV}_{1}$ decline $\mathrm{ml} / \mathrm{yr}$ & $0.5(-50.0-170.0)$ & $60.0(-30.0-170.0)$ & 0.335 \\
\hline Frequent $\mathrm{AE} n,(\%)$ & 7 (10.0\%) & $12(20.3 \%)$ & 0.099 \\
\hline \multicolumn{4}{|l|}{ Inflammatory markers } \\
\hline IL-6 (pg/dl) & $1.46(0.43-2.46)$ & $2.67(1.52-6.80)$ & $<0.001^{*}$ \\
\hline $\mathrm{ESR}(\mathrm{mm} / \mathrm{h})$ & $10.00(6.00-16.25)$ & $15.00(7.00-21.00)$ & $0.011^{*}$ \\
\hline $\mathrm{CRP}(\mathrm{mg} / \mathrm{dl})$ & $1.25(0.63-2.30)$ & $3.39(1.49-6.74)$ & $0.044^{*}$ \\
\hline
\end{tabular}

Data are presented as median (interquartile range) for continuous variables and number (percentage) for categorical variables.

*This P-value was analyzed by ANCOVA (after adjusting age, BMI, smoking, and underlying diseases).

$A E$, acute exacerbation; BMI, body mass index; CAT, chronic obstructive pulmonary disease assessment test; CRP, C-reactive protein; ESR, erythrocyte sedimentation rate; $D L_{C O}$, diffusing capacity of the lung for carbon monoxide; $F E V_{1}$, forced expiratory volume in 1 s; FVC, forced vital capacity; IL-6, interleukin-6; mMRC, modified Medical Research Council; S-COPD, smoking-induced chronic obstructive pulmonary disease; SGRQ, St. George Respiratory Questionnaire; T-COPD, tuberculosis-associated COPD.

candidate metabolites. We performed global metabolomics profiling, which can screen thousands of unknown features in two conditions. The detected metabolic features were then subjected to statistical analyses. Supplementary Figure 1 shows the results of the unsupervised pattern recognition analysis. Supplementary Figures 1A-C shows the score plots describing variation and account for the varied influences of the NC and S-COPD groups, the NC and T-COPD groups, and the TCOPD and S-COPD groups. In the univariate analysis, FDRadjusted values $<0.05$ with a 2.0 -fold change were applied for marker selection. Sixty-nine metabolites remained after the univariate analysis compared the NC and S-COPD groups, and 58 metabolites remained after the comparison for the NC and T-COPD groups. Among these, 29 metabolites overlapped and their changes (increase or decrease) showed similar trends (Figure 2A). Volcano plots of each analysis are available in Supplementary Figure 2.

For further analysis, targeted metabolic profiling was performed, which measures the absolute concentrations of 


\section{Cohort}

Normal control $(n=39)$

S-COPD $(n=70)$

T-COPD $(n=59)$

\section{Clinical tests \\ Lung function test \\ Clinical examinations}

\section{Statistical} analysis

Univariate analysis Multivariate analysis Correlation analysis (with clinical parameters)

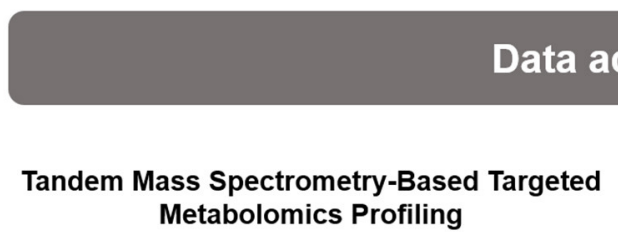
Metabolomics Profiling

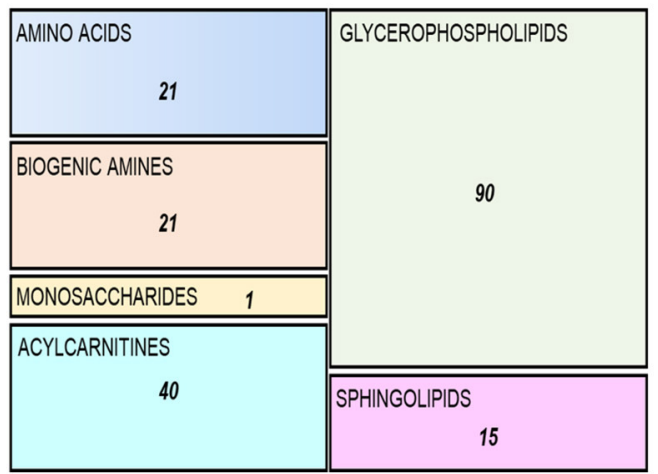

\section{Quadrupole Time-Of-Flight Mass Spectrometry- Based Global Metabolomics Profiling}

FIGURE 1 | Schematic of the study. This study included 39 healthy subjects, 70 patients with S-COPD, and 59 patients with T-COPD. All patients underwent clinical examinations, including lung function tests and metabolic profiling. For the targeted analysis, a tandem mass spectrometry-based technique was used. The absolute quantification of 188 metabolites, including 21 amino acids, 21 biogenic amines, one monosaccharide, 40 acylcarnitines, 90 glycerophospholipids, and 15 sphingolipids, was performed. For global metabolomic profiling, a QTOF-MS-based technique was chosen. Metabolic biomarkers were selected by performing multivariate and univariate analyses. The correlations of the metabolic biomarkers with clinical parameters were then calculated. NC, normal control; T-COPD, TB-associated COPD; S-COPD, smoking-associated COPD.

\section{A Untargeted analysis}

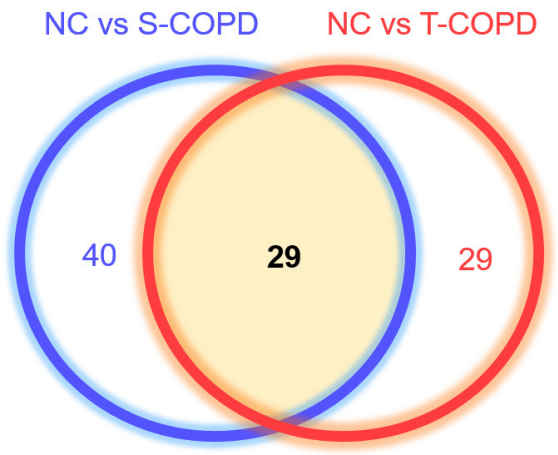

B Targeted analysis

\section{NC vs S-COPD NC vs T-COPD}

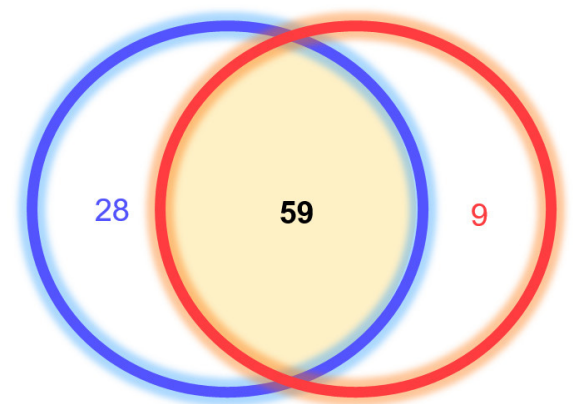

FIGURE 2 | Venn diagram of metabolites identified in patients with T-COPD and S-COPD. (A) Comparison of the NC and S-COPD groups revealed that 8,183 ions and 69 metabolites were significantly altered. In the comparison of the NC and T-COPD groups, 5,193 ions were detected, and 58 metabolites were significantly different. Twenty-nine metabolites were shared by the two COPD groups. (B) Using the targeted analysis, 188 metabolites were quantified, 87 metabolites were significantly altered between the NC and S-COPD groups, and 68 metabolites were significantly altered between the NC and T-COPD groups. Fifty-nine metabolites overlapped in these two comparisons and their direction of regulation was also similar in the S-COPD and T-COPD groups, compared to the NC group. The blue circle indicates metabolic biomarkers obtained from the comparison of the NC and S-COPD groups, and the red circle represents metabolic biomarkers identified in the comparison of the NC and T-COPD groups. NC, normal control; T-COPD, TB-associated COPD; S-COPD, smoking-associated COPD.

molecules and the conversion rates from one molecule to another. For the targeted analysis, 188 metabolites were quantified based on the LC-MS analyses. This technique provided quantitative data for 40 acylcarnitines, 42 amino acids, biogenic amines, monosaccharides, 15 sphingolipids, and 90 glycerophospholipids in each sample. The absolute 
concentration in each sample was then adjusted for statistical analysis, with a $P$-value $<0.05$, indicating significance. We obtained 87 distinct metabolites that differentiated the NC and $\mathrm{S}-\mathrm{COPD}$ groups, and 68 metabolites that differentiated the NC and T-COPD groups (Figure 2B).

Significantly altered metabolites are displayed in Figure 3. All the identified metabolites were mapped and linked to each other based on their biological interactions. In patients with SCOPD, we observed increased levels of long-chain fatty acids (specifically myristoleic acid, palmitic acid, and oleic acid), bile acid-related metabolites, and multiple glycerophospholipids and acylcarnitines (Figure 3A and Supplementary Table 3). All metabolites identified using global metabolomics are listed in Supplementary Table 4 . In the T-COPD group, we observed specific changes in the arachidonic and eicosanoic acid metabolic pathways (Figure 3A and Supplementary Table 3). Similar changes in the levels of myristoleic acid, palmitic acid, oleic acid, ethyl arachidonate, and prostaglandin E, were observed in all the groups. However, there was more than a two-fold increase in the levels of arachidonic acid, eicosanoic acid, docosapentaenoic acid, dihomo-alpha-linolenic acid, dihomo-linoleic acid, hydroxyeicosatrienoic acid (HETrE), and hydroxyeicosapentaenoic acid (HEPE) in the T-COPD patients, compared to the healthy controls, whereas no gradual changes were observed in the S-COPD group.

\section{Changes in Tryptophan Catabolism}

Among the 188 targeted metabolites subjected to absolute quantification, 59 were shared among the COPD groups and predominantly comprised acylcarnitines or glycerophospholipids. Notably, biogenic amines (kynurenine and serotonin) and amino acids (aspartate and threonine) were detected in the T-COPD group. Furthermore, the association between tryptophan catabolism and disease status was investigated (Figure 4). Although the total serum concentration of tryptophan did not change among the groups, we observed significant alterations in the kynurenine and serotonin levels in T-COPD patients (Figures $\mathbf{4 A}, \mathbf{C}$ ). Along with the changes in metabolite levels, the enzymatic activities of indoleamine dioxygenase (IDO) and tryptophan hydroxylase (TPH) were altered in the T-COPD group, which showed increased IDO activity compared with the control group (Figure 4D), and decreased TPH activity (Figure 4E). These changes were reversed in the S-COPD group. Although the total tryptophan level did not change, tryptophan catabolizing enzyme activities differed according to disease status, particularly in the T-COPD group. A categorical analysis was subsequently performed to determine whether the levels of these metabolites changed with disease severity (Supplementary Figure 3). Patients in each group were divided based on a FEV 1 cut-off value of $50 \%$. None of these markers, including tryptophan, kynurenine, serotonin, and IDO/TPH activity, were related to lung function. Thus, these metabolic biomarkers of T-COPD are unique phenotypes in the COPD population; however, they still have a limited ability to identify disease severity. Figure 5 reveals the distinct metabolic pathways that were specifically altered in patients with T-COPD. After excluding the metabolites that overlapped with those identified in patients with S-COPD, metabolic pathways related to arachidonic and eicosanoic acid, bile acid metabolism, and tryptophan catabolism, were identified in this study.

\section{Discrimination of T-COPD and S-COPD by Targeted Metabolites}

We further performed logistic regression of discrimination of T-COPD and S-COPD for targeted metabolites. Aspartate was the important metabolites associated with T-COPD diagnosis and serotonin and asparagine were the important metabolites associated with S-COPD (Supplementary Table 5). When we perform ROC analysis with aspartate, asparagine and serotonin, the AUC of T-COPD diagnosis was 0.780 (Supplementary Figure 4).

\section{DISCUSSION}

This is the first study to comprehensively profile serum samples from patients with COPD caused by smoking or a previous TB infection. The levels of IL-6 and CRP, and the SGRQ scores, were found to be higher in patients with T-COPD than in those with S-COPD. The multi-platform approach further discovered endogenous metabolites that distinguished these two disease groups. The metabolism of fatty acids, especially arachidonic acid and eicosanoic acid, and tryptophan catabolism were correlated, particularly in the T-COPD group. These results are consistent with the findings of our previous study, demonstrating an association between systemic inflammation and the COPD subphenotype in this cohort (16).

Overall, the metabolites identified in patients with T-COPD and S-COPD shared some common features, as shown in Figure 2. The presence of similar physiological symptoms such as dyspnea, sputum production, and acute exacerbation might result in similar metabolic changes, even though the etiology of S-COPD and T-COPD are different. We observed major changes in the levels of acylcarnitines and phospholipids in both COPD groups, with the levels being higher than that in healthy subjects. The close relationship between acylcarnitines and physiological activities is well-known as the evidence of energy metabolism. Circulating fatty acids are generally conjugated with carnitines to be utilized as an energy source in mitochondria, and their use is decreased in damaged lungs (17). Thus, increased serum acylcarnitine levels can be expected in COPD, and altered phospholipid levels could be another metabolic hallmark of COPD. The imbalance between phospholipids and lysophospholipids in COPD is controversial. However, multiple studies have reported a close association between increased levels of circulating phospholipids and the production of proinflammatory mediators during COPD exacerbations (18).

Changes in fatty acid metabolites indicate notable distinction between T-COPD and S-COPD. Here, the T-COPD group showed higher levels of some lipid metabolites, such as arachidonic acid or eicosanoic acids, than the S-COPD group did. Arachidonic acid, a polyunsaturated fatty acid (PUFA), is a member of the $\omega-6$ fatty acid family and the primary 


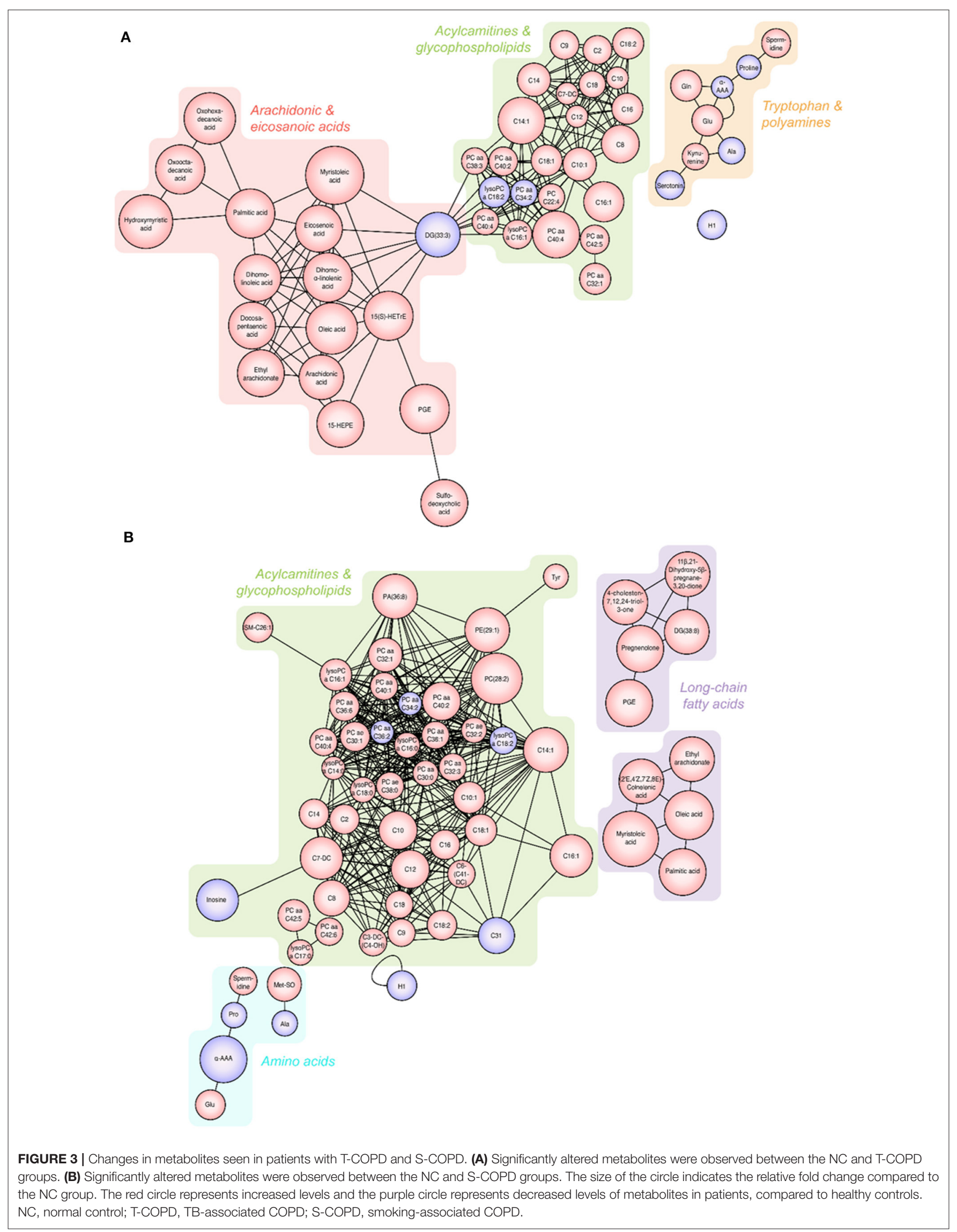


A

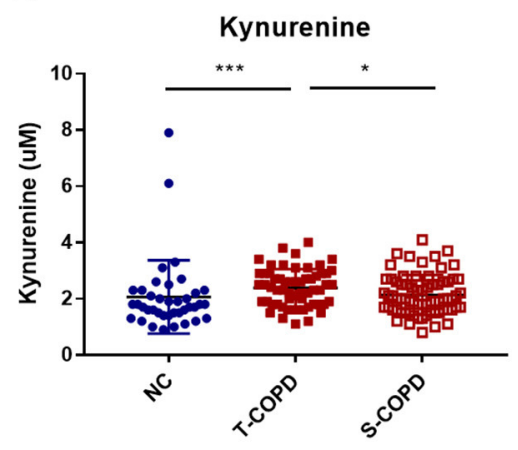

C

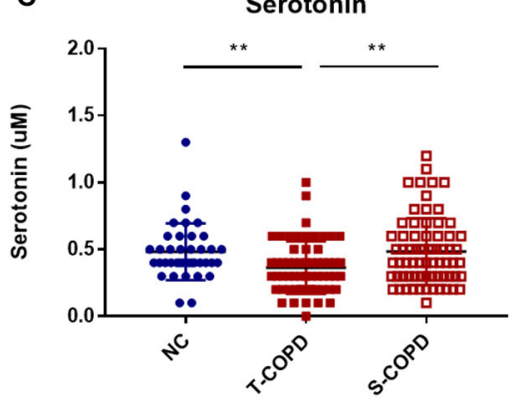

B

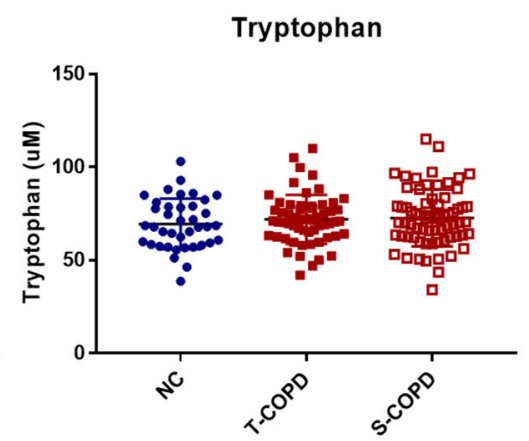

D

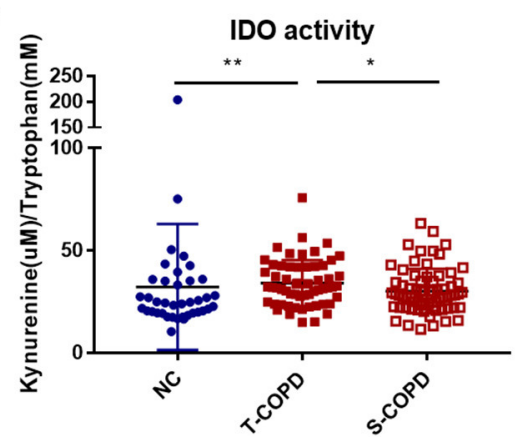

E

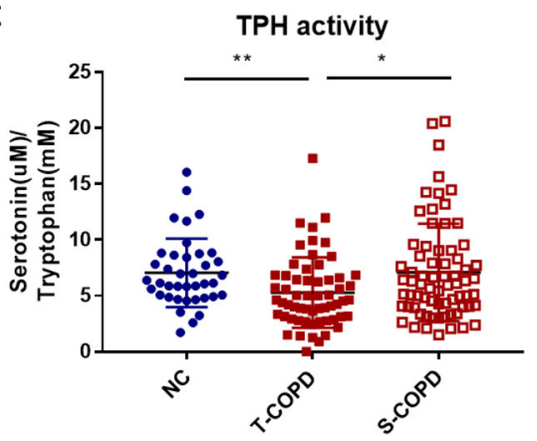

FIGURE 4 | Alternation of tryptophan metabolism in patients with T-COPD. Serum concentrations of (A) kynurenine, (B) tryptophan, and (C) serotonin are shown in scatter plots. (D) and (E) indicate IDO and TPH activities, respectively. Data are presented as the mean \pm standard error of the mean. ${ }^{*} P$-value $<0.05$, ${ }^{* *} P$-value $<0.01$, and ${ }^{* * *} P$-value < 0.001. NC, normal control; T-COPD, TB-associated COPD; S-COPD, smoking-associated COPD; IDO, indoleamine dioxygenase; TPH, tryptophan hydroxylase.

target of cyclooxygenases (COX) and lipoxygenase (LOX). Byproducts such as prostaglandins and eicosanoids, well-known proinflammatory mediators, are generated following oxidization (17). Lipid metabolism in the lungs is associated with chronic respiratory failure, airway obstruction, bronchoconstriction, IL-6 generation, vascular permeability, airway remodeling, mucus secretion, and inflammation in COPD (19). Interestingly, excess levels of arachidonic acid and eicosanoids were observed in the TCOPD group. Additionally, oxylipins were positively correlated with IL-6 and CRP levels, and docosapentanoic acid, 15-HEPE, and long-chain fatty acids were associated with worsening lung function and acute exacerbation. As lipid metabolite levels were increased in both disease groups compared to the controls, being significantly higher in the T-COPD group than in the $\mathrm{S}$-COPD group, T-COPD can be considered to have similar pathogenesis as S-COPD, but causes more severe local and systemic inflammation.

Moreover, tryptophan catabolism showed greater activation in the T-COPD group than in the S-COPD group. Tryptophan catabolism through the IDO pathway exerts antimicrobial and anti-inflammatory effects in certain infectious or inflammatory conditions $(20,21)$. Increased levels of inflammatory cytokines such as IL-6 induce IDO expression, and IDO induces regulatory $\mathrm{T}$ cells to regulate inflammatory responses (22). The effect of tryptophan catabolism on inflammation is known; several studies have already analyzed IDO and TPH activities in COPD patients. Although a study reported increased IDO activity during acute exacerbations of COPD (21), no apparent alterations in this biological process have been reported to date $(23,24)$. Similar to the results from previous studies, the levels of the associated metabolites were altered in the T-COPD group, but not in the S-COPD group. Furthermore, higher systemic levels of inflammatory mediators, such as IL-6 and CRP, were observed in the T-COPD group than in the S-COPD group. Based on these findings, coexisting bronchiectasis, destroyed lung parenchyma, and bacterial colonization may cause repeated infections and inflammation $(25,26)$. Activation of immunological mechanisms may also result in sustained systemic inflammation after TB, and elevated IL-6 levels may subsequently induce activation of the IDO pathway.

The contribution of the gut microbiota cannot be excluded. Tryptophan is a microbiome-derived amino acid catalyzed by three pathways: (1) direct conversion into ligands of the aryl hydrocarbon receptor via the gut microbiota; (2) entry into the IDO-mediated kynurenine pathway in immune and epithelial cells; and (3) serotonin production by tryptophan hydroxylase in enterochromaffin cells (27, 28). Although the role of functional microbiota in the pathophysiology 


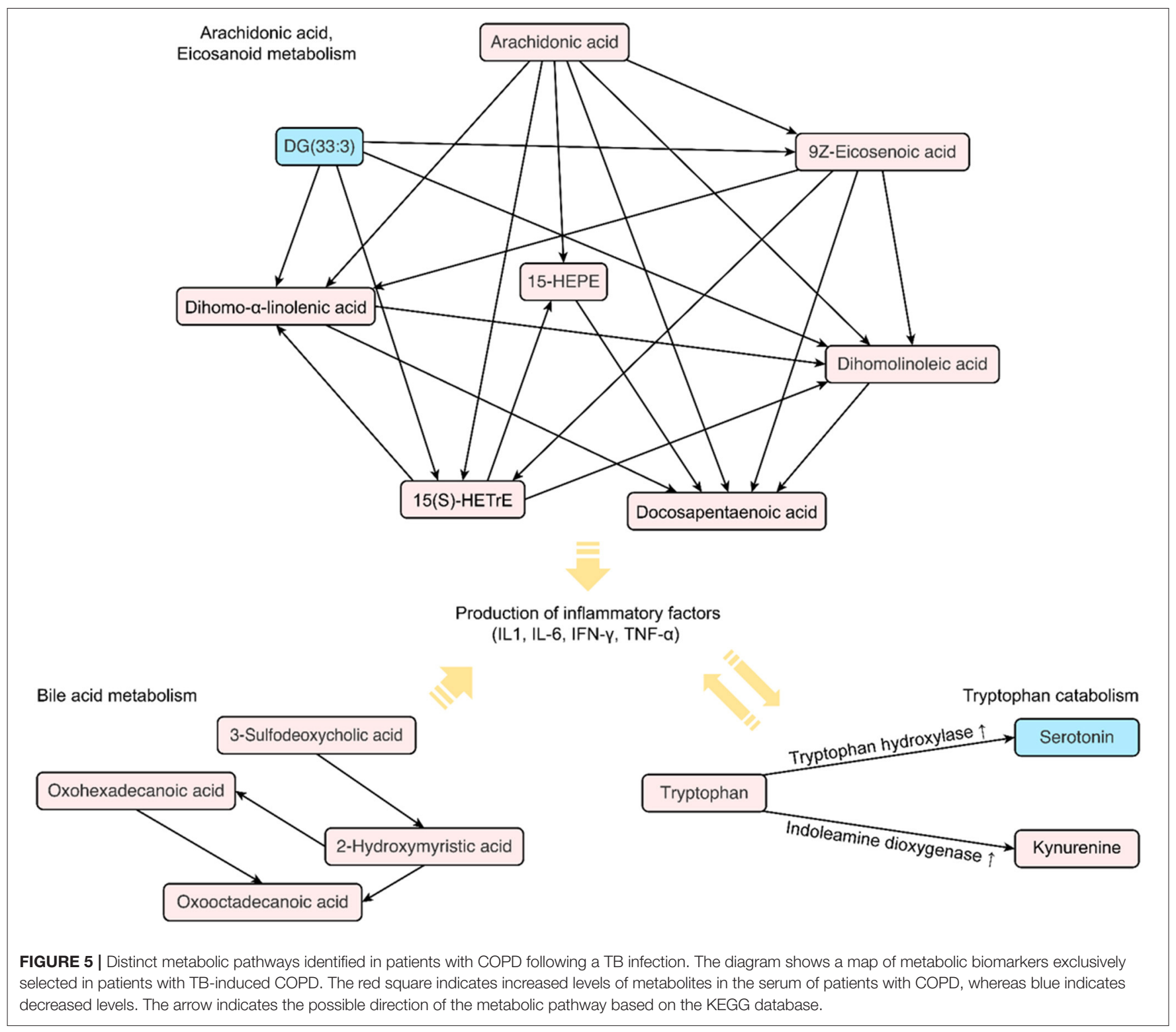

of COPD is being investigated, alterations in the gut microbiome have been observed; its diversity decreases with increasing disease severity, compared to healthy subjects (29, 30). Nevertheless, the relationship between tryptophan catabolism and COPD is controversial, although the role of TB infection in tryptophan catabolism is well-known. According to previous in vivo and in vitro studies, $\mathrm{TB}$ induces potent activation of IDO, thereby inducing tryptophan catabolism for kynurenine production, which predominantly occurs in lung and immune cells $(29,31)$. IDO activity suppression has been shown to improve clinical symptoms of lung destruction and immune response in experimental studies (29). Although T-COPD patients were completely cured of TB, there could have been some irreversible continuous pathophysiological changes. Therefore, these patients might experience persistent effects of the resolved TB infection throughout their lifetime.
Smoking increases systemic neutrophil infiltration and sequesters leukocytes in lung capillaries by decreasing their deformability (32). Factors directly modulating the host immune system increase the production of inflammatory mediators such as interleukins, tumor necrosis factor-alpha (TNF- $\alpha$ ), and $\mathrm{CRP}$, resulting in tissue damage $(33,34)$. The irreversible destruction of alveolar walls induces a progressive decline in lung function and increases inflammation (2, 35). Thus, various therapies for S-COPD, including inhaled corticosteroids, bronchodilators (including $\beta 2$-agonists and anticholinergics), theophyllines, and phosphodiesterase- 4 inhibitors, are targeted to reduce airway inflammation $(36,37)$. Although recent studies have focused on anti-inflammatory treatment for S-COPD (38), the importance of managing the inflammatory features of T-COPD is usually underestimated. Considering that patients with T-COPD exhibited increased inflammatory responses that might be linked with fatty acid pathways and tryptophan 
catabolism, further studies should focus on treatment strategies to control the associated inflammatory consequences of TBinduced COPD. Following the treatment for TB, aggressive treatments for controlling inflammation should be considered along with the existing COPD treatment modalities, for patients with T-COPD.

This study has some limitations. First, the strengths of associations between the metabolic biomarkers and demographic parameters in healthy controls were not performed due to the absence of pulmonary function tests and cytokine measurements. Correlation analysis of metabolic biomarkers and clinical parameters was conducted only among COPD patients (Supplementary Figure 5). Consequently, we found a positive correlation between oxylipins and IL-6 and CRP levels, while diacylglyceride and colnelenic acid levels were negatively correlated with these parameters. Additionally, positive correlations were identified between lung function scores, such as the rate of $\mathrm{FEV}_{1}$ decline and acute exacerbation, and the levels of docosapentanoic acid, 15-HEPE, and longchain fatty acids were identified. However, the absence of healthy control information resulted in a relatively small slope of the correlations. Second, the metabolomics data included only male patients, since the prevalence of S-COPD is remarkably low in Korean women (39). A validation study would require examinations of females to improve the generalizability of our findings. Moreover, we performed metabolic profiling within one batch and selected metabolites based on strict cut-off values, the FDR-adjusted $P$-value, and fold change, to minimize the output size of raw data and to avoid the batch effect. These processes might limit our ability to detect large changes in metabolites in the direct comparison of patients with S-COPD and those with T-COPD. Further examinations using a validation cohort are required to determine the reproducibility of our findings.

In conclusion, this is the first study to demonstrate increased levels of several inflammatory metabolites in patients with T-COPD compared to those with SCOPD. Lipid and tryptophan metabolism could be potential therapeutic targets for COPD, particularly in T-COPD.

\section{REFERENCES}

1. Mathers CD, Loncar D. Projections of global mortality and burden of disease from 2002 to 2030. PLoS Med. (2006) 3:e442. doi: 10.1371/journal.pmed.0030442

2. Barnes PJ, Burney PG, Silverman EK, Celli BR, Vestbo J, Wedzicha JA, et al. Chronic obstructive pulmonary disease. Nat Rev Dis Primers. (2015) 1:15076. doi: 10.1038/nrdp.2015.76

3. Ravimohan S, Kornfeld H, Weissman D, Bisson GP. Tuberculosis and lung damage: from epidemiology to pathophysiology. Eur Respir Rev. (2018) 27:170077. doi: 10.1183/16000617.0077-2017

4. Yakar HI, Gunen H, Pehlivan E, Aydogan S. The role of tuberculosis in COPD. Int J Chron Obstruct Pulmon Dis. (2017) 12:323-9. doi: 10.2147/COPD.S116086

\section{DATA AVAILABILITY STATEMENT}

The metabolomics data are available in the electronic Supplementary Material and at the NIH Common Fund's National Metabolomics Data Repository (NMDR) website (Project ID: PR000943).

\section{ETHICS STATEMENT}

The studies involving human participants were reviewed and approved by the institutional review boards (Korea University Guro Hospital: KUGH 13246; Seoul St. Mary's Hospital: KC15OIMI0553; and Chonbuk National University Hospital: 2015-01-018-005), and it fulfilled the tenets of the Declaration of Helsinki. The patients provided written informed consent. The patients/participants provided their written informed consent to participate in this study.

\section{AUTHOR CONTRIBUTIONS}

DK, JO, and J-YC conceptualized the study. JO, CR, SP, and JS collected samples. DK performed metabolic profiling. The results were interpreted by $\mathrm{DK}$, JO, and J-YC. The original draft preparation was done by DK and JO. J-YC reviewed the manuscript. Funding acquisition was prepared by JO and J-YC. All authors contributed to the article and approved the submitted version.

\section{FUNDING}

This study was supported by the Bio and Medical Technology Development Programme of the National Research Foundation (NRF) funded by the Korean government (MSIT) (Grant Numbers 2019M3E5D3073365 and 2019M3E5D1A01068994).

\section{SUPPLEMENTARY MATERIAL}

The Supplementary Material for this article can be found online at: https://www.frontiersin.org/articles/10.3389/fmed. 2021.619077/full\#supplementary-material
5. Allwood BW, Myer L, Bateman ED. A systematic review of the association between pulmonary tuberculosis and the development of chronic airflow obstruction in adults. Respiration. (2013) 86:76-85. doi: 10.1159/000350917

6. Byrne AL, Marais BJ, Mitnick CD, Lecca L, Marks GB. Tuberculosis and chronic respiratory disease: a systematic review. Int J Infect Dis. (2015) 32:138-46. doi: 10.1016/j.ijid.2014.12.016

7. Lee JH, Chang JH. Lung function in patients with chronic airflow obstruction due to tuberculous destroyed lung. Respir Med. (2003) 97:123742. doi: 10.1016/S0954-6111(03)00255-5

8. Sarkar M, Srinivasa, Madabhavi I, Kumar K. Tuberculosis associated chronic obstructive pulmonary disease. Clin Respir J. (2017) 11:28595. doi: $10.1111 /$ crj.12621

9. Radovic M, Ristic L, Ciric Z, Dinic-Radovic V, Stankovic I, Pejcic T, et al. Changes in respiratory function impairment following the treatment of severe 
pulmonary tuberculosis-limitations for the underlying COPD detection. Int $J$ Chron Obstruct Pulmon Dis. (2016) 11:1307-16. doi: 10.2147/COPD.S106875

10. Malherbe ST, Shenai S, Ronacher K, Loxton AG, Dolganov G, Kriel M, et al. Persisting positron emission tomography lesion activity and Mycobacterium tuberculosis mRNA after tuberculosis cure. Nat Med. (2016) 22:1094100. doi: $10.1038 / \mathrm{nm} .4177$

11. Reinke SN, Gallart-Ayala H, Gómez C, Checa A, Fauland A, Naz S, et al. Metabolomics analysis identifies different metabotypes of asthma severity. Eur Respir J. (2017) 49:1601740. doi: 10.1183/13993003.01740-2016

12. Kim DJ, Yoon S, Ji SC, Yang J, Kim YK, Lee S, et al. Ursodeoxycholic acid improves liver function via phenylalanine/tyrosine pathway and microbiome remodelling in patients with liver dysfunction. Sci Rep. (2018) 8:11874. doi: 10.1038/s41598-018-30349-1

13. Kim DJ, Cho EJ, Yu KS, Jang IJ, Yoon JH, Park T, et al. Comprehensive Metabolomic Search for Biomarkers to Differentiate Early Stage Hepatocellular Carcinoma from Cirrhosis. Cancers. (2019) 11:1497. doi: 10.3390/cancers11101497

14. Oh JY, Lee YS, Min KH, Hur GY, Lee SY, Kang KH, et al. Increased urinary 1-histidine in patients with asthma-COPD overlap: a pilot study. Int J Chron Obstruct Pulmon Dis. (2018) 13:1809-18. doi: 10.2147/COPD.S163189

15. Kim DJ, Chung H, Ji SC, Lee S, Yu KS, Jang IJ, et al. Ursodeoxycholic acid exerts hepatoprotective effects by regulating amino acid, flavonoid, and fatty acid metabolic pathways. Metabolomics. (2019) 15:30. doi: 10.1007/s11306-019-1494-5

16. Oh JY, Lee YS, Min KH, Hur GY, Lee SY, Kang KH, et al. Difference in systemic inflammation and predictors of acute exacerbation between smoking-associated COPD and tuberculosis-associated COPD. Int J Chron Obstruct Pulmon Dis. (2018) 13:3381-7. doi: 10.2147/COPD.S177371

17. Chen H, Li Z, Dong L, Wu Y, Shen H, Chen Z. Lipid metabolism in chronic obstructive pulmonary disease. Int J Chron Obstruct Pulmon Dis. (2019) 14:1009-18. doi: 10.2147/COPD.S196210

18. Bowler RP, Jacobson S, Cruickshank C, Hughes GJ, Siska C, Ory DS, et al. Plasma sphingolipids associated with chronic obstructive pulmonary disease phenotypes. Am J Respir Crit Care Med. (2015) 191:27584. doi: 10.1164/rccm.201410-17710C

19. Ran N, Pang Z, Gu Y, Pan H, Zuo X, Guan X, et al. An updated overview of metabolomic profile changes in chronic obstructive pulmonary disease. Metabolites. (2019) 9:111. doi: 10.3390/metabo90 60111

20. Takikawa O. Biochemical and medical aspects of the indoleamine 2, 3-dioxygenase-initiated L-tryptophan metabolism. Biochem Biophys Res Commun. (2005) 338:12-9. doi: 10.1016/j.bbrc.2005. 09.032

21. Gulcev M, Reilly C, Griffin TJ, Broeckling CD, Sandri BJ, Witthuhn BA, et al. Tryptophan catabolism in acute exacerbations of chronic obstructive pulmonary disease. Int J Chron Obstruct Pulmon Dis. (2016) 11:243546. doi: 10.2147/COPD.S107844

22. Suzuki Y, Suda T, Yokomura K, Suzuki M, Fujie M, Furuhashi $\mathrm{K}$, et al. Serum activity of indoleamine 2,3-dioxygenase predicts prognosis of community-acquired pneumonia. J Infect. (2011) 63:215-22. doi: 10.1016/j.jinf.2011.07.003

23. Labaki WW, Gu T, Murray S, Curtis JL, Yeomans L, Bowler RP, et al. SPIROMICS metabolomics study. Sci Rep. (2019) 9:11367. doi: 10.1038/s41598-019-47761-w

24. Naz S, Bhat M, Ståhl S, Forsslund H, Sköld CM, Wheelock ÅM, et al. Dysregulation of the Tryptophan Pathway Evidences Gender Differences in COPD. Metabolites. (2019) 9:212. doi: 10.3390/metabo9100212

25. Jordan TS, Spencer EM, Davies P. Tuberculosis, bronchiectasis and chronic airflow obstruction. Respirology. (2010) 15:6238. doi: 10.1111/j.1440-1843.2010.01749.x
26. Mao B, Lu HW, Li MH, Fan LC, Yang JW, Miao XY, et al. The existence of bronchiectasis predicts worse prognosis in patients with COPD. Sci Rep. (2015) 5:10961. doi: 10.1038/srep10961

27. Agus A, Planchais J, Sokol H. Gut microbiota regulation of tryptophan metabolism in health and disease. Cell Host Microbe. (2018) 23:71624. doi: 10.1016/j.chom.2018.05.003

28. Zelante T, Iannitti RG, Cunha C, De Luca A, Giovannini G, Pieraccini G, et al. Tryptophan catabolites from microbiota engage aryl hydrocarbon receptor and balance mucosal reactivity via interleukin-22. Immunity. (2013) 39:372-85. doi: 10.1016/j.immuni.2013.08.003

29. Huang YJ, Erb-Downward JR, Dickson RP, Curtis JL, Huffnagle GB, Han MK. Understanding the role of the microbiome in chronic obstructive pulmonary disease: principles, challenges, and future directions. Transl Res. (2017) 179:71-83. doi: 10.1016/j.trsl.2016.06.007

30. Martinez FJ, Erb-Downward JR, Huffnagle GB. Significance of the microbiome in chronic obstructive pulmonary disease. Ann Am Thorac Soc. (2013) 10(Suppl):S170-9. doi: 10.1513/AnnalsATS.201306-204AW

31. Blumenthal A, Nagalingam G, Huch JH, Walker L, Guillemin GJ, Smythe GA, et al. Tuberculosis induces potent activation of IDO-1, but this is not essential for the immunological control of infection. PLoS ONE. (2012) 7:e37314. doi: 10.1371/journal.pone.0037314

32. MacNee W, Wiggs B, Belzberg AS, Hogg JC. The effect of cigarette smoking on neutrophil kinetics in human lungs. N Engl J Med. (1989) 321:9248. doi: 10.1056/NEJM198910053211402

33. de Moraes MR, da Costa AC, Correa Kde S, Junqueira-Kipnis AP, Rabahi MF. Interleukin- 6 and interleukin- 8 blood levels' poor association with the severity and clinical profile of ex-smokers with COPD. Int J Chron Obstruct Pulmon Dis. (2014) 9:735-43. doi: 10.2147/COPD.S64135

34. Terashima T, Wiggs B, English D, Hogg JC, van Eeden SF. Phagocytosis of small carbon particles (PM10) by alveolar macrophages stimulates the release of polymorphonuclear leukocytes from bone marrow. Am J Respir Crit Care Med. (1997) 155:1441-7. doi: 10.1164/ajrccm.155.4.9105091

35. O'Toole RF, Shukla SD, Walters EH. TB meets COPD: An emerging global co-morbidity in human lung disease. Tuberculosis. (2015) 95:65963. doi: 10.1016/j.tube.2015.08.005

36. Cosio BG, Iglesias A, Rios A, Noguera A, Sala E, Ito $\mathrm{K}$, et al Low-dose theophylline enhances the anti-inflammatory effects of steroids during exacerbations of COPD. Thorax. (2009) 64:424-9. doi: 10.1136/thx.2008.103432

37. Mirza S, Clay RD, Koslow MA, Scanlon PD. COPD Guidelines: a review of the 2018 GOLD report. May Clin Proc. (2018) 93:14881502. doi: 10.1016/j.mayocp.2018.05.026

38. Barnes PJ. New anti-inflammatory targets for chronic obstructive pulmonary disease. Nat Rev Drug Discov. (2013) 12:543-59. doi: 10.1038/nrd4025

39. Lee JY, Chon GR, Rhee CK, Kim DK, Yoon HK, Lee JH, et al. Characteristics of patients with chronic obstructive pulmonary disease at the first visit to a pulmonary medical Center in Korea: The KOrea COpd Subgroup Study Team Cohort. J Korean Med Sci. (2016) 31:553-60. doi: 10.3346/jkms.2016.31.4.553

Conflict of Interest: The authors declare that the research was conducted in the absence of any commercial or financial relationships that could be construed as a potential conflict of interest.

Copyright $\odot 2021 \mathrm{Kim}, \mathrm{Oh}$, Rhee, Park, Shim and Cho. This is an open-access article distributed under the terms of the Creative Commons Attribution License (CC BY). The use, distribution or reproduction in other forums is permitted, provided the original author(s) and the copyright owner(s) are credited and that the original publication in this journal is cited, in accordance with accepted academic practice. No use, distribution or reproduction is permitted which does not comply with these terms. 